ORIGINAL ARTICLE

\title{
Dopamine Alters Tactile Perception in Parkinson's Disease
}

\author{
Aimee J. Nelson, Azra Premji, Navjot Rai, Tasnuva Hoque, Mark Tommerdahl, \\ Robert Chen
}

\begin{abstract}
Background: Abnormal somatosensory processing may contribute to motor impairments observed in Parkinson's disease (PD). Dopaminergic medications have been shown to alter somatosensory processing such that tactile perception is improved. In PD, it remains unclear whether the temporal sequencing of tactile stimuli is altered and if dopaminergic medications alter this perception. Methods: Somatosensory tactile perception was investigated using temporal order judgment in patients with Parkinson's disease on and off dopaminergic medications and in aged-matched healthy controls. Measures of temporal order judgment were acquired using computer controlled stimulation to digits 2 and 3 on the right hand and subjects were required to determine which stimuli occurred first. Two experimental tasks were compared, temporal order judgment without and with synchronization whereby digits 2 and 3 were vibrated synchronously in advance of the temporal order judgment sequence of stimuli. Results: Temporal order judgment in PD patients off and on medications were similar to controls. Temporal order judgment preceded by synchronous vibration impaired tactile acuity in controls and in PD off medications to similar degrees, but this perceptual impairment by synchronous vibration was not present in PD patients on medications. Conclusions: These findings suggest that dopamine in PD reduces cortico-cortical connectivity within SI and this leads to changes in tactile sensitivity.
\end{abstract}

RÉSUMÉ: La dopamine altère la perception tactile dans la maladie de Parkinson. Contexte : Le traitement anormal de l'information somatosensitive pourrait contribuer à l'altération de la motricité observée dans la maladie de Parkinson (MP). Il a été démontré que les médicaments dopaminergiques modifient le traitement de l'information somatosensitive de telle sorte que la perception tactile en est améliorée. Dans la MP, nous ne savons pas si la séquence temporelle des stimuli tactiles est modifiée et si les médicaments dopaminergiques modifient cette perception. Méthode : La perception tactile somatosensitive a été étudiée au moyen du test de discernement de l'ordre de succession de stimuli chez des patients atteints de la MP avec et sans effet de la médication dopaminergique et chez des témoins en bonne santé appariés pour l'âge. L'évaluation du discernement de l'ordre temporel de stimuli a été effectuée au moyen de la stimulation contrôlée par ordinateur des $2 \mathrm{e}$ et $3 \mathrm{e}$ doigts de la main droite. Les sujets devaient déterminer quel stimulus était appliqué le premier. Deux tâches expérimentales ont été comparées : le discernement de l'ordre temporel sans et avec synchronisation, soit l'application au préalable d'une vibration synchrone aux les 2e et 3e doigts. Résultats : Le discernement de l'ordre temporel chez les patients atteints de la MP sans et avec l'effet des médicaments était semblable à celui des témoins. Lorsque le test de discernement de l'ordre temporel était précédé par les vibrations synchrones, l'acuité tactile chez les témoins et les patients sans l'effet de la médication était perturbée à des degrés semblables, mais cette perturbation perceptuelle due aux vibrations synchrones n'était pas présente chez les patients sous l'effet de la médication. Conclusions : Ces constatations suggèrent que, chez les patients atteints de la MP, la dopamine diminue la connectivité cortico-corticale dans l'aire somatosensitive SI, ce qui provoque des changements dans la sensibilité tactile.

Can. J. Neurol. Sci. 2012; 39: 52-57

Individuals with Parkinson's disease (PD) frequently have sensory symptoms and somatosensory abnormalities that may contribute to the motor symptoms ${ }^{1}$. In PD, tactile localization ${ }^{2}$ and spatial acuity ${ }^{3}$ are impaired. Deficiencies in tactile processing may relate to a loss of epidermal nerve fibers and Meissner corpuscles ${ }^{4}$, alterations in cortical circuitry targeted by nigro-striatal output ${ }^{5}$, changes in somatosensory afferent transmission $^{6}$ or mutation of the PINK1 gene ${ }^{7}$. In PD, tactile acuity in the time domain has been studied using temporal discrimination threshold (TDT), which refers to the minimum time interval between two sequential stimuli to be perceived as distinct. Compared to controls, TDT in PD is increased on the hand $^{8}$ and foot ${ }^{9}$. Anatomical substrates that underpin TDT include the pre-supplementary motor area, anterior cingulate cortex, primary and posterior parietal cortex, striatum and thalamus ${ }^{10,11}$. Tactile acuity in the time domain may also be assessed using temporal order judgment (TOJ), defined as the minimal interstimulus interval necessary to detect the temporal order of two sequential stimuli delivered at distinct locations on the skin. Temporal order judgment is different from TDT because it requires participants to determine the order of the

From the Department of Kinesiology (AJN, AP, NR), University of Waterloo, Waterloo; Division of Neurology (AJN, TH, RC) and Krembil Neuroscience Centre, Toronto Western Research Institute, Toronto, Ontario, Canada; Biomedical Engineering (MT), University of North Carolina, Chapel Hill, North Carolina, USA.

Received May 25, 2011. Final Revisions SubmitTed July 14, 2011.

Correspondence to: Aimee J. Nelson, University of Waterloo, 200 University Ave. W., BMH 1116, Waterloo, Ontario, N2L 3G1, Canada. 
arrival of stimuli applied to multiple skin sites. Further, compared to TDT, TOJ emphasizes higher-order abilities related to learning and memory ${ }^{12}$. Similar to TDT, TOJ may be sensitive to disturbances of the basal ganglia ${ }^{11}$. A previous study found that TOJ was increased in PD patients with homozygous PINK1 mutation as well as asymptomatic heterozygous PINK1 mutation carriers $^{12}$. However, TOJ in non-genetic PD remains to be examined.

In healthy individuals, TOJ may be selectively impaired by adding a weak synchronous vibrating stimulus in advance of the sequential test stimuli ${ }^{13}$. The perceptual impairment is thought to result from the synchronization of neural ensembles within primary somatosensory cortex (SI) that respond to two skin sites stimulated during TOJ. The co-activation of the two ensembles by the synchronous vibrating stimulus perceptually binds the two skin sites thereby making it difficult to identify the site that received the first stimulus within the TOJ pair ${ }^{13,14}$. The neural mechanism mediating the synchronization effect remains unclear although gamma-Aminobutyric acid (GABA)ergic inhibition occurring between SI mini-columns, the smallest unit of columnar organization within the neocortex consisting of 80-100 neurons that span layers $\mathrm{II}-\mathrm{VI}^{15}$, is a likely candidate and is important for preserving temporal acuity for optimal sensorimotor control.

Dopamine (DA) projections originating in the ventral tegmental area terminate in the neocortex ${ }^{16}$ including $\mathrm{SI}^{17}$, suggesting a potential role for DA in somatosensation. The DA system is suggested to influence cortico-cortical connectivity and higher-order integrative processes ${ }^{18}$. In PD, DA medications improve $\mathrm{TDT}^{8,9,19}$. Percepts relying on minicolumn connectivity such as the synchronization TOJ effect may be altered by DA inputs. The present study compared TOJ with and without synchronization in PD patients in the on and off medication states and healthy controls. We hypothesized that TOJ thresholds would be higher in PD versus controls similar to the observations made for TDT measures $8,9,20$. Owing to the perceptual improvements associated with dopaminergic medications, we further predicted that TOJ would be improved in the 'on' versus the 'off' states in PD with and without synchronization stimuli.

\section{METHODS}

\section{Subjects}

Nine patients with PD (mean age 59 years, range 47 to 72 years, 2 women, Table) and ten healthy controls (mean age 56 years, range 41 to 73 years, 5 women) were studied. Parkinson's disease patients were recruited from the Toronto Western Hospital Movement Disorders Clinic from August 2009 to February 2010. Two of the nine patients were left-handed and all controls were right-handed as measured using a subset of the Oldfield Handedness Inventory ${ }^{21}$. Parkinson's disease patients were tested in two sessions separated by at least one week. Overnight withdrawal of dopaminergic medications was required for the practically defined 'off' session and testing was performed in the morning following withdrawal. Regular

Table: Patient demographics

\begin{tabular}{c|c|c|c|c|c|c|c}
\hline $\mathbf{P T}$ & $\begin{array}{c}\text { Age } \\
\text { (yrs) }\end{array}$ & Gender & Handedness & $\begin{array}{c}\text { Most } \\
\text { Affected } \\
\text { Hand }\end{array}$ & $\begin{array}{c}\text { Symptom } \\
\text { Duration } \\
\text { (years) }\end{array}$ & $\begin{array}{c}\text { Medication } \\
\text { (LEs) }\end{array}$ & $\begin{array}{c}\text { UPDRS III } \\
\text { (off/on) }\end{array}$ \\
\hline 1 & 61 & $\mathrm{M}$ & $\mathrm{R}$ & $\mathrm{R}$ & 4 to 5 & 700 (L-DOPA, \\
PRA) & $8.0 / 5.0$ \\
\hline 2 & 72 & $\mathrm{M}$ & $\mathrm{R}$ & $\mathrm{L}$ & 4 & 562.5 (L-DOPA) & $24 / 16.5$ \\
\hline 3 & 57 & $\mathrm{M}$ & $\mathrm{R}$ & $\mathrm{L}$ & 5 & 560 (L-DOPA, & $10.0 / 8.0$ \\
\hline 4 & 65 & $\mathrm{M}$ & $\mathrm{R}$ & $\mathrm{R}$ & 1 & 300 (L-DOPA) & $25.5 / 21.5$ \\
\hline 5 & 56 & $\mathrm{M}$ & $\mathrm{R}$ & $\mathrm{L}$ & 10 & $620(\mathrm{~L}-\mathrm{DOPA}$, & $15.5 / 16.5$ \\
\hline 6 & 52 & $\mathrm{M}$ & $\mathrm{R}$ & $\mathrm{R}$ & 8 & 600 (L-DOPA) & $11.5 / 4.5$ \\
\hline 7 & 71 & $\mathrm{~F}$ & $\mathrm{~L}$ & $\mathrm{~L}$ & 10 & 600 (L-DOPA) & $27.5 / 13.5$ \\
\hline 8 & 58 & $\mathrm{~F}$ & $\mathrm{~L}$ & $\mathrm{~L}$ & 5 & 900 (PRA) & $12.0 / 3.0$ \\
\hline 9 & 47 & $\mathrm{M}$ & $\mathrm{R}$ & $\mathrm{R}$ & 10 & 900 (L-DOPA, & $23 / 5.5$ \\
\hline
\end{tabular}

Medications are converted into levodopa equivalents using the following formula: levodopa equivalent (LE) $=$ total dose of regular levodopa (with peripheral decarboxylase inhibitor $)+(0.75 \mathrm{x}$ dose of controlled-release levodopa $)+(100 \mathrm{x}$ dose of pramipexole $)+(16.7 \mathrm{x}$ dose of ropinirole $)+(0.2$ $\mathrm{x}$ dose of entacapone). Medications are abbreviated as follows: L-DOPA = levodopa; $\mathrm{ROP}=$ ropinirole; $\mathrm{PRA}=$ pramipexole; $\mathrm{ENT}=\mathrm{entacapone}$; $\mathrm{PT}$ = patient $\mathrm{m}=$ male; $\mathrm{f}=$ female; $\mathrm{r}=$ right; 1 = left. Unified Parkinson's Disease Rating Scale (UPDRS) motor section (items 18-31). 
medication timing and dosages were administered for the 'on' session and testing was performed within two to four hours following intake. The order of 'on' and 'off' testing was counterbalanced across participants. All participants provided written informed consent. The UPDRS III was used to assess PD motor signs. The University Health Network Research Ethics Board approved the study and conformed to the Declaration of Helsinki.

\section{Temporal Order Judgment}

The Cortical Metrics (CM) Device ${ }^{13,22}$ was used to collect TOJ data. The right hand was supinated and positioned beneath the two contact probes of the CM device. The volar surfaces of the second and third digit tip (index and middle finger) received tactile stimulation. Each trial began with the probes indented until an initial force of $0.1 \mathrm{~g}$ was registered by the device. To ensure even probe contact with the digit pads, the probes were indented an additional $500 \mu \mathrm{m}$. Each testing session began with practice trials whereby participants had to meet the criteria of performing five consecutive trials correctly. During practice trials visual feedback was displayed on a laptop computer (a happy face and sad face for correct and incorrect performance, respectively). No feedback was given during the subsequent test trials.

For TOJ, vibrotactile stimuli were delivered to the fingertips of the second and third digits at a constant intensity $(200 \mu \mathrm{m}$ amplitude), frequency $(25 \mathrm{~Hz})$ and duration (40 ms) using a forced-choice paradigm. The initial inter-stimulus interval (ISI) between the sequential vibrotactile stimuli was set at $150 \mathrm{~ms}$ and was adjusted by a set step-size of $15 \mathrm{~ms}$ depending on the accuracy of the participant's response. The participant was queried to identify which finger received the first stimulus by using a mouse click. Using the left hand to indicate their response selection, a left mouse click indicated vibration

A

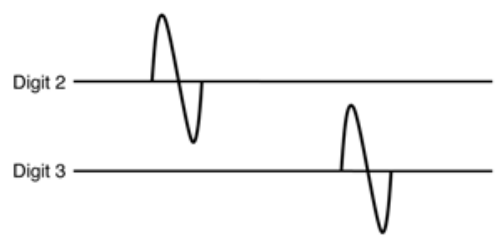

B

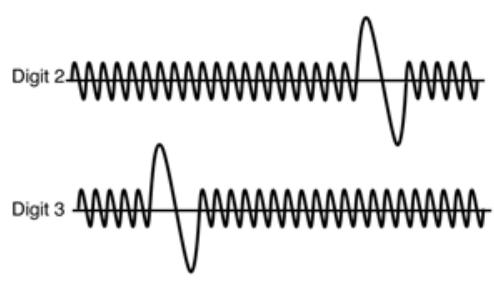

Figure 1: Temporal order judgment task. A. TOJ task displaying sequential vibrotactile stimulation of digits two and three separated by a given interstimulus interval. $B$. TOJ synchronization task whereby the TOJ task is preceded by synchronized low amplitude vibration of both digits. occurred on right hand second digit and a right mouse click indicated vibration occurred on right hand third digit. The ISI was adjusted with a one up/one down protocol for the first 20 trials and two up /one down protocol for the remainder of trials (i.e. two correct choices decreased the ISI by the set step size (15 $\mathrm{ms}$ ) while one incorrect choice increased the ISI by the set step size). The duration of each trial, consisting of the two pulses and respective ISI was one second with a five second inter-trial interval. The order of pulse delivery was randomized between the two fingertips for each trial. Figure 1A displays a schematic of the TOJ task. For TOJ synchronization, the identical protocol outlined in Figure 1A was delivered but with the addition of constant $5 \mu \mathrm{m}$ stimulation synchronized across both fingertips for 380 to $485 \mathrm{~ms}$ in advance of the TOJ stimuli. The synchronized stimuli were presented for a total of one second. Figure 1B displays a schematic of the TOJ synchronization task. A total of 40 trials were performed for each of the TOJ and TOJ synchronization tasks. The TOJ threshold for both tasks was defined as the average of the last five trials (i.e. trials 36 through 40) as used elsewhere ${ }^{13,14}$.

\section{Data Analysis}

To compare task performance between PD and controls, two separate two-way repeated measures analysis of variance (ANOVA) were performed (one for PD 'on' and one for PD 'off') using within-subject factors TASK (two levels; TOJ, TOJ synchronized) and between-subject factor GROUP (two levels; controls, PD). Post-hoc Tukey's tests were used to identify significance in the event of significant main effects or interactions. To investigate the effect of dopaminergic medication within PD, a two-way repeated measures ANOVA was performed using within-subject factors TASK (two levels; TOJ, TOJ synchronized) and DRUG (two levels; on, off). Significance for each ANOVA was set at $\mathrm{p}<0.05$. Post-hoc Tukey's tests were used to identify significance in the event of significant main effects or interactions and were Bonferroni corrected for the number of comparisons (four comparisons, $\mathrm{p}<$ 0.012). The four comparisons were synchronization TOJ versus TOJ in the 'on' and 'off' states and 'on' versus 'off' for synchronization TOJ and TOJ.

\section{RESULTS \\ PD versus Controls}

Two-way ANOVA comparing PD 'off' versus controls revealed a main effect of TASK $\left(\mathrm{F}_{(1,17)}=6.95, \mathrm{p}=0.01\right)$ and no effect of GROUP $(\mathrm{p}=0.69)$ or GROUP by TASK interaction $(p=0.96)$. The group data is shown in Figure 2. Temporal order judgment synchronization impaired tactile performance in both controls and PD 'off' medication to similar degrees. The magnitude of the effect in controls is similar to a previous study $^{13}$. Two-way ANOVA comparing PD 'on' medication versus controls revealed no significant effect of TASK $(p=0.09)$, GROUP $(\mathrm{p}=0.58)$ or their interaction $(\mathrm{p}=0.27)$.

\section{$P D$ - on versus off dopaminergic medications}

The two-way ANOVA comparing PD 'off' versus 'on' medications revealed a significant main effect of DRUG 


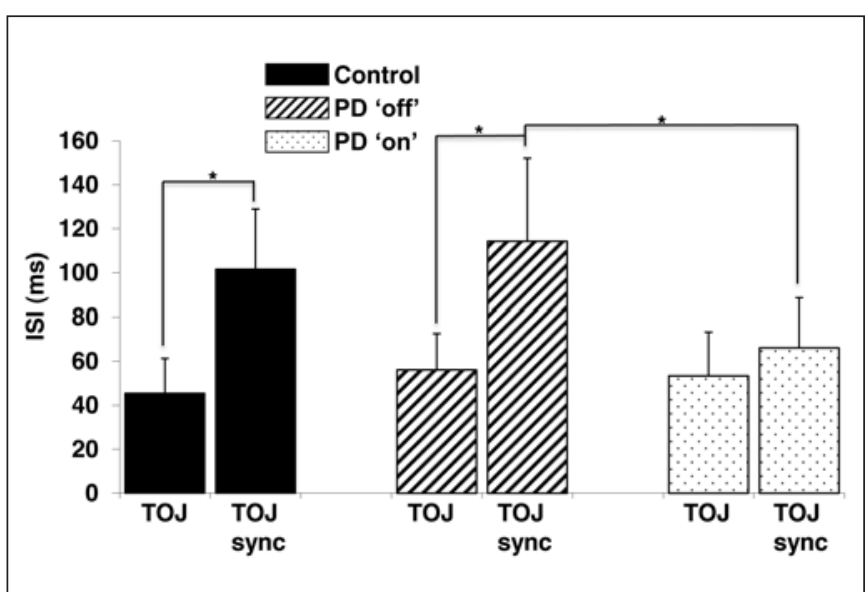

Figure 2: TOJ in controls, $P D$ 'off' and 'on' dopaminergic medications. Group-averaged TOJ (with standard errors) in controls (black bars), PD 'off' (hatched bars) and PD 'on'(dotted bars) dopaminergic medications during TOJ and TOJ with synchronous (sync) $25 \mathrm{~Hz}$ stimulation applied to the digits. Synchronous stimulation impairs TOJ performance in controls and PD 'off' medication but not in the 'on' state. Post-hoc Tukey's tests revealed that TOJ synchronization impairs performance in controls and PD 'off' (main effect). Within PD, significant differences were found between 'on' and 'off' medication during synchronous stimulation and between tasks in the 'off' state only. * indicates $p<0.05$.

$\left(\mathrm{F}_{(1,8)}=5.43, \mathrm{p}=0.04\right)$ and interaction between DRUG and TASK $\left(\mathrm{F}_{(1,8)}=6.31, \mathrm{p}=0.03\right)$ with a nearly significant effect of TASK $\left(\mathrm{F}_{(1,8)}=4.77, \mathrm{p}=0.06\right)$. Post-hoc comparison revealed that TOJ synchronization impaired performance in the 'off' state (synchronization TOJ versus TOJ; $\mathrm{p}=0.001$ ) similar to the controls. However, in the 'on' state TOJ synchronization did not impair performance $(\mathrm{p}=0.34)$. Further, performance during TOJ synchronization was significantly improved in the 'on' versus 'off' state $(\mathrm{p}=0.005)$.

\section{DISCUSSION}

The experiments presented are the first to investigate TOJ in non-genetic PD patients. Temporal order judgment in PD patients on and off dopaminergic medications were similar to aged-matched controls. Using a synchronization paradigm that diminishes TOJ performance in controls, similar effects could be obtained in PD in the off state. However, in the presence of dopaminergic medications, the synchronization effect was not observed. The data suggest that dopaminergic medications alter the neural substrates that underpin the mechanisms that mediate synchronized neural activity between cortical minicolumns within SI. The present findings, implications in PD and suggestions on the effects of dopaminergic medications to modulate tactile perception are discussed.

Dopaminergic medications appear to improve impaired tactile temporal perception in PD. For example, somatic, visual and auditory TDT were partially restored after a single dose of L-dopa ${ }^{8}$ and L-dopa improves TDT on the hand ${ }^{19}$ and foot ${ }^{9}$ to near normal values. In PD patients with subthalamic nucleus (STN) deep brain stimulation (DBS), switching the DBS on worsened TDT in the medication on state but STN DBS had no effect in medication off state ${ }^{19}$. In the present study, we observed that dopaminergic medications do not improve TOJ though thresholds were not different from controls. However, DA medications prevented the normal decrement in performance observed with synchronous stimuli applied to adjacent digits (i.e. no synchronization effect), thereby supporting a role for DA in modulating tactile temporal perception. One prediction that arises from the present study is that controls would fail to show the synchronization effect following intake of dopaminergic medications, similar to that seen in PD 'on' and also similar to the effects of DA on tactile coactivation effects ${ }^{23}$. The tactile coactivation paradigm involves vibrating the digit tip for three hours to produce an improvement in spatial acuity of the stimulated area $^{24}$. Administration of $100 \mathrm{mg}$ of L-dopa in healthy controls blocks the effects of the tactile coactivation paradigm and therefore prevents non-associative learning ${ }^{23}$. It may be that DA in healthy individuals will also block the TOJ synchronization effect.

In healthy individuals, synchronously applied tactile stimuli to adjacent digits is thought to lead to synchronized co-activation of nearby cortical ensembles (minicolumns) within SI that receive that input ${ }^{13}$. In healthy individuals, synchronized activation of minicolumns leads to perceptual binding such that the identity of the temporal order of stimuli is degraded ${ }^{13}$. In addition to SI, temporal discrimination is associated with neural responses in secondary somatosensory cortex, supplementary motor area, prefrontal cortex, inferior parietal lobule, basal ganglia, and the cerebellum ${ }^{10,11,25,26}$. It is unclear to what extent these loci and the spinal cord and thalamus may contribute to the synchronization effect. However, in patients with autism, the synchronization effect is not present ${ }^{14}$. In autism, the neuropil space that largely encompasses GABAergic interneurons is reduced $^{27}$ and deficiencies in GABA are present ${ }^{28}$ supporting a role for GABA in mediating synchronous activity between minicolumns. In the present study, we noted that PD patients 'on' medications behave similarly to patients with autism and do not demonstrate the synchronization effect. This effect in the 'on' state may result if DA has a net inhibitory influence on GABAergic interneurons that mediate the synchronous activity between minicolumns within SI. Recent evidence suggests that DA antagonists acting at D1 and D2-type receptors in SI in rats increased the amplitude of somatosensory evoked potentials and increase cortical excitability ${ }^{29}$. Data from the present study suggests that DA acts to increase SI excitability in PD, possibly via inhibition of local inhibitory interneurons between minicolumns.

Studies using transcranial magnetic stimulation (TMS) have also yielded insight into the mechanisms of DA within SI. Compared to single pulse TMS, paired-pulse TMS over SI reduces the perception of electrical stimuli delivered to the finger when the time delay between the two pulses is 10 or 15 ms, likely via activation of local intracortical inhibitory circuits $^{30}$. However, a shorter delay between pairs of TMS pulses increases the perception of peripheral stimuli in controls and in PD off medication. Changes in perception are not observed in PD on medication ${ }^{31}$ suggesting that DA specifically modifies intracortical circuitry within SI. Collectively, the latter study and the present results suggest that exogenous DA alters neural 
processing within SI.

In Fiorio et al, ${ }^{12} \mathrm{PD}$ patients show impairments in TOJ following withdrawal from medication compared to controls $(\sim 150 \mathrm{~ms}$ in PD versus $\sim 55 \mathrm{~ms}$ in controls). In contrast, the present study demonstrated that TOJ in PD off medication is not significantly different from controls (56 ms in PD versus $45 \mathrm{~ms}$ in controls). The disparity between the two studies likely reflects differences in methodological approaches. First, Fiorio et a ${ }^{12}$ use electrical stimuli that can activate a larger skin surface compared to the small focus of mechanical stimuli used in the present study (5 $\mathrm{mm}$ diameter probes). The spatial spread of electrical versus mechanical stimuli may not yield differences in control participants but may be pronounced in PD where there is a loss of epidermal nerve fibers ${ }^{4}$ and the sensation of electrical stimuli relies on inputs arriving from a larger surface of skin. Second, although both studies used suprathreshold stimuli to perform TOJ measures it is unclear how electrical intensities compare with those derived from mechanical stimulation. In the present study indentation amplitude was set at $200 \mu \mathrm{m}$ to remove any issues related to stimulus detection that could potentially impair the ability to make TOJ judgments. Last, temporal thresholds may be influenced by the psychophysical method used. Fiorio et $\mathrm{al}^{12}$ use a method of ascending limits and define TOJ as the first of three consecutive ISIs at which correct performance occurs. In contrast, we used a staircase method to adjust the ISI in an up and down manner ${ }^{32}$ until performance plateaus. The difference in psychophysical approaches may also explain the greater TOJ Fiorio et al ${ }^{12}$ measured in their control group $(\sim 55 \mathrm{~ms})$ versus our study $(45 \mathrm{~ms})$. An alternative explanation for the disparity between studies may relate to the differences between PINK1 PD and non-genetic PD; TOJ impairments in PINK1 PD may be different from those found in non-genetic PD.

L-dopa may exert different and opposite effects on cortical excitability within the primary motor cortex (M1) compared to SI. For example, the reduced M1 intra-cortical inhibition in PD is restored following administration of dopaminergic medications ${ }^{33}$, although it has been suggested that reduced cortical inhibition in PD is due to increased cortical facilitation rather than decreased GABAergic inhibition ${ }^{34}$. Despite the differential net effects of DA in M1 versus SI, it appears that the net behavioral effect is one of improvement such that movement initiation is improved and tactile performance is not degraded. However, DA medication is also shown to reduce of short latency afferent inhibition ${ }^{35}$, and impairs other forms of somatic sensations such as proprioception that may relate to motor symptoms ${ }^{36}$. L-dopa clearly improves the motor symptoms in PD but the present study demonstrates that this occurs at the expense of altering the cortico-cortical connectivity within the somatosensory system. Although the effect of such altered activity does not appear to be detrimental to daily tactile function, DA medications nonetheless alter cortical dynamics within SI and may therefore manifest as subtle changes in sensory and motor behavior.

\section{ACKNOWLEDGEMENTS}

Dr. Nelson is supported by the Natural Science and Engineering Research Council of Canada. N. Rai is supported by an Ontario Graduate Scholarship. Dr. Chen is supported by the Catherine Manson Chair in Movement Disorders.

\section{REFERENCES}

1. Shin HW, Kang SY, Sohn YH. Dopaminergic influence on disturbed spatial discrimination in Parkinson's disease. Mov Disord. 2005; 20: 1640-3.

2. Schneider JS, Diamond SG, Markham CH. Deficits in orofacial sensorimotor function in Parkinson's disease. Ann Neurol. 1986; 19: $275-82$

3. Sathian K, Zangaladze A, Green J, Vitek JL, DeLong MR. Tactile spatial acuity and roughness discrimination: impairments due to aging and Parkinson's disease. Neurology. 1997; 49: 168-77.

4. Nolano M, Provitera V, Estraneo A, et al. Sensory deficit in Parkinson's disease: evidence of a cutaneous denervation. Brain. 2008; 131: 1903-11.

5. Purves D, Augustine GJ, Fitzpatrick D, et al. Modulation of movement by the basal ganglia. Sunderland, MA: Sinauer Associates; 2008.

6. Rossini PM, Babiloni F, Bernardi G, et al. Abnormalities of shortlatency somatosensory evoked potentials in parkinsonian patients. Electroencephalogr Clin Neurophysiol. 1989; 74: 277-89.

7. Gierthmuhlen J, Lienau F, Maag R, et al. Somatosensory processing in a German family with PINK1 mutations: its potential role in Parkinson disease. J Neurol Neurosurg Psychiatry. 2009; 80: 571-4.

8. Artieda J, Pastor MA, Lacruz F, Obeso JA. Temporal discrimination is abnormal in Parkinson's disease. Brain. 1992; $115 \mathrm{Pt} 1$ : 199-210.

9. Lee MS, Kim HS, Lyoo CH. "Off" gait freezing and temporal discrimination threshold in patients with Parkinson disease. Neurology. 2005; 64: 670-4.

10. Pastor MA, Day BL, Macaluso E, Friston KJ, Frackowiak RS. The functional neuroanatomy of temporal discrimination. J Neurosci. 2004; 24: 2585-91.

11. Lacruz F, Artieda J, Pastor MA, Obeso JA. The anatomical basis of somaesthetic temporal discrimination in humans. J Neurol Neurosurg Psychiatry. 1991; 54: 1077-81.

12. Fiorio M, Valente EM, Gambarin $M$, et al. Subclinical sensory abnormalities in unaffected PINK1 heterozygotes. J Neurol. 2008; 255: 1372-7.

13. Tommerdahl M, Tannan V, Zachek M, Holden JK, Favorov OV. Effects of stimulus-driven synchronization on sensory perception. Behav Brain Funct. 2007; 3: 61.

14. Tommerdahl M, Tannan V, Holden JK, Baranek GT. Absence of stimulus-driven synchronization effects on sensory perception in autism: evidence for local underconnectivity? Behav Brain Funct. 2008; 4: 19.

15. Mountcastle VB. Perceptual neuroscience, the cerebral cortex. Cambridge, MA and London, England: Harvard University Press; 1998.

16. Smythies J. Section II. The dopamine system. Int Rev Neurobiol. 2005; 64: 123-72.

17. Descarries L, Lemay B, Doucet G, Berger B. Regional and laminar density of the dopamine innervation in adult rat cerebral cortex. Neuroscience. 1987; 21: 807-24.

18. Foote SL, Morrison JH. Extrathalamic modulation of cortical function. Annu Rev Neurosci. 1987; 10: 67-95.

19. Conte A, Modugno N, Lena F, et al. Subthalamic nucleus stimulation and somatosensory temporal discrimination in Parkinson's disease. Brain. 2010; 133: 2656-63.

20. Lee MS, Lyoo CH, Lee MJ, Sim J, Cho H, Choi YH. Impaired finger dexterity in patients with parkinson's disease correlates with discriminative cutaneous sensory dysfunction. Mov Disord. 2010; 25: 2531-5.

21. Oldfield RC. The assessment and analysis of handedness: the Edinburgh inventory. Neuropsychologia. 1971; 9: 97-113.

22. Tannan V, Dennis RG, Zhang Z, Tommerdahl M. A portable tactile sensory diagnostic device. J Neurosci Methods. 2007; 164: 131-8.

23. Bliem B, Frombach E, Ragert P, et al. Dopaminergic influences on changes in human tactile acuity induced by tactile coactivation. Exp Brain Res. 2007; 181: 131-7. 
24. Godde B, Stauffenberg B, Spengler F, Dinse HR. Tactile coactivation-induced changes in spatial discrimination performance. J Neurosci. 2000; 20: 1597-604.

25. de Lafuente V, Romo R. Neural correlate of subjective sensory experience gradually builds up across cortical areas. Proc Natl Acad Sci USA. 2006; 103: 14266-71.

26. Rao SM, Mayer AR, Harrington DL. The evolution of brain activation during temporal processing. Nat Neurosci. 2001; 4: 317-23.

27. Casanova MF, Buxhoeveden DP, Switala AE, Roy E. Minicolumnar pathology in autism. Neurology. 2002; 58: 428-32.

28. Belmonte MK, Allen G, Beckel-Mitchener A, Boulanger LM, Carper RA, Webb SJ. Autism and abnormal development of brain connectivity. J Neurosci. 2004; 24: 9228-31.

29. Hosp JA, Hertler B, Atiemo CO, Luft AR. Dopaminergic modulation of receptive fields in rat sensorimotor cortex. Neuroimage. 2011; 54: 154-60.

30. Koch G, Franca M, Albrecht UV, Caltagirone C, Rothwell JC. Effects of paired pulse TMS of primary somatosensory cortex on perception of a peripheral electrical stimulus. Exp Brain Res. 2006; 172: 416-24.
31. Palomar FJ, Diaz-Corrales F, Carrillo F, Fernandez-Del-Olmo M, Koch G, Mir P. Sensory perception changes induced by transcranial magnetic stimulation over the primary somatosensory cortex in Parkinson's disease. Mov Disord. Epub 2011 Jun 2.

32. Gescheider G. Chapter 3: The classical psychophysical methods. Psychophysics: the fundamentals. Mahway, New Jersey, USA: Lawrence Erlbaum Associates; 1997.

33. Ridding MC, Sheean G, Rothwell JC, Inzelberg R, Kujirai T. Changes in the balance between motor cortical excitation and inhibition in focal, task specific dystonia. J Neurol Neurosurg Psychiatry. 1995; 59: 493-8.

34. MacKinnon CD, Gilley EA, Weis-McNulty A, Simuni T. Pathways mediating abnormal intracortical inhibition in Parkinson's disease. Ann Neurol. 2005; 58: 516-24.

35. Sailer A, Molnar GF, Paradiso G, Gunraj CA, Lang AE, Chen R. Short and long latency afferent inhibition in Parkinson's disease. Brain. 2003; 126: 1883-94.

36. Mongeon D, Blanchet P, Messier J. Impact of Parkinson's disease and dopaminergic medication on proprioceptive processing. Neuroscience. 2009; 158: 426-40. 